\title{
Fabrication of a SERS Substrate of Gold Nanoparticles by Dewetting
}

\author{
I.A. Estrada-Moreno ${ }^{1}$, R.B. Dominguez-Cruz ${ }^{1}$, V.C. Osuna-Galindo ${ }^{1}$, J.T. Holguín-Momaca ${ }^{2}$, A. Vega- \\ Ríos $^{2}$, A. Márquez-Lucero ${ }^{3}$ \\ 1. CONACyT - Centro de Investigación en Materiales Avanzados, S.C., Miguel de Cervantes 120, \\ 31136, Chihuahua, Chihuahua, México. \\ 2. Centro de Investigación en Materiales Avanzados, S.C. Chihuahua, México, 31136. \\ 3. Centro de Investigación en Materiales Avanzados, S.C. Durango, México 30147.
}

Metal nanoparticle arrays continue to attract attention because of their unique and functional properties compared to their macroscopic counterpart. The main interest in these structures is related to their specific optical properties [1]. When noble metal nanostructures, such as gold or silver, are illuminated by electromagnetic radiation they exhibit an optical phenomenon known as Surface Plasmon Resonance (SPR) and is caused by the coherent oscillation of conduction band electrons in the nanostructure. The electromagnetic field in the near area of the nanostructure of the metal also shows specific properties when electromagnetic waves radiate the system in conditions of resonance. The enormous extension of this electromagnetic field is used in one of its most important applications, the surface-enhanced Raman Scattering (SERS), which is one of the most powerful spectroscopic techniques and has been widely used in some areas such as chemistry, materials science, and biosciences [2].

The gold nanoparticles were obtained by a simple two steps method, the sputtering deposition of a film of gold, $5 \mathrm{~nm}$, and $10 \mathrm{~nm}$, on a silicon substrate and its thermal dewetting. The obtained substrates were characterized by the scanning electron microscope and Raman measurements. Figure 1 shows the gold nanoparticles for both films, $5 \mathrm{~nm}(\mathrm{Au} 5 \mathrm{~nm})$ and $10 \mathrm{~nm}$ (Au10nm). The mean diameter of the nanoparticles is $50.12 \mathrm{~nm}$ for the Au5nm and $115.02 \mathrm{~nm}$ for the Au10nm. The smaller particles should have a better performance as substrate SERS according to with reported in the literature. Also, we can observe in the inset the uniform distribution of the nanoparticles.

The Raman spectroscopy measurements were carried out in a Raman confocal microspectrometer with the $632.8 \mathrm{~nm}$ line. First, the absorption of Rhodamine and the silicon substrate were measured. Then, a drop of Rhodamine solution $(210 \mu \mathrm{M})$ was deposited onto the surface of the SERS substrate. After dry, the measurements were made. Rhodamine presents characteristic signal about 1200, 1380, 1500 and $1650 \mathrm{~cm}^{-1}$, in the presence of nanoparticles, i.e., the SERS effect.

Figure 2a shows the spectra of pure silicon and Rhodamine; it is observed that raman spectra of Rhodamine is weak in the absence of nanoparticles. In Figure $2 b$, there is a noticeable increase in the intensity of the spectra for Rhodamine on the gold substrates at different thicknesses. The substrate in which were deposited a film of $5 \mathrm{~nm}$ of thickness shows a greater increase in the spectra as it is known that the properties of SPR depend on the shape, size, arrangement of the nanoparticles and the dielectric constant of the medium surrounding the nanoparticles [3].

In summary, we fabricated a SERS substrate by a simple method, a thermal dewetting of a thin film of gold. The Raman spectra showed that the SERS substrates that can be used reliably for the detection of analytes at low concentrations $(210 \mu \mathrm{M})$. Also, it was corroborated than the smaller size of particles, the better improve in SERS effect, as the SERS substrate with $50 \mathrm{~nm}$ diameter showed better enhacement. 


\section{References:}

[1] Z Li, M Yoshino, and A Yamanaka, Procedia CIRP 5 (2013), p. 42-46.

[2] R Nikov, N Nedyalkov, P Atanasov, D Hirsch, B Rauschenbach, K Grochowska, and G Sliwinski, Applied Surface Science, 374 (2015) p. 36-41.

[3] T Phuc, M Yoshino,, AYamanaka, and T Yamamoto, Procedia CIRP 5 (2013), p. 47-52.

[4] The authors thank to Wilber Antunez and Pedro Piza for their helpful assistance to this work.

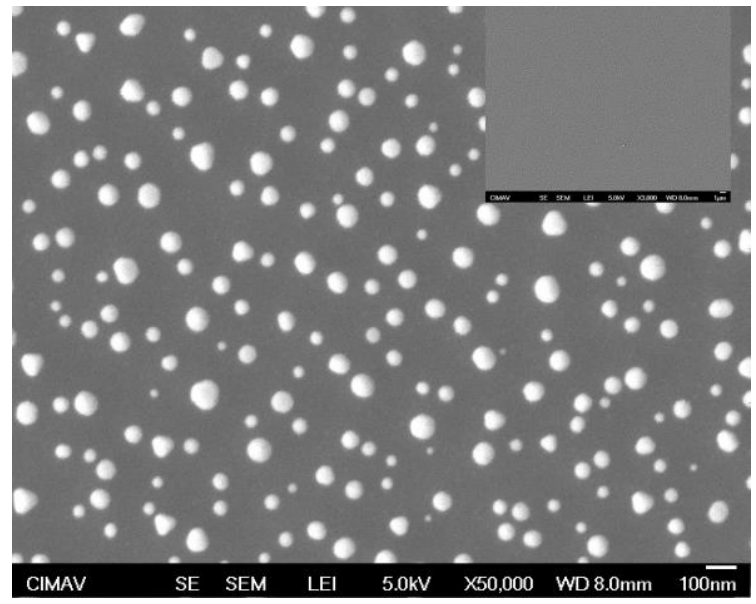

a)

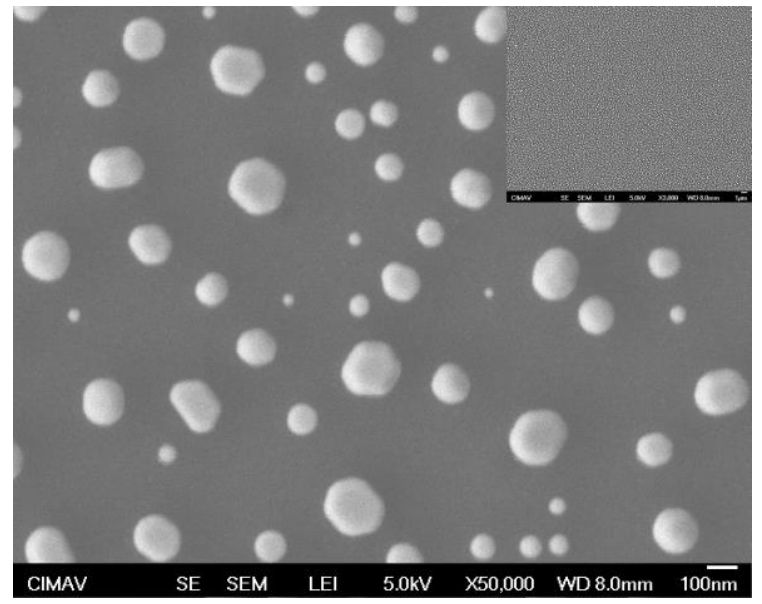

b)

Figure 1. SEM micrographs of gold nanoparticles obtained by thermal dewetting, a) from a gold film of $5 \mathrm{~nm}$ and b) a gold film of $10 \mathrm{~nm}$.

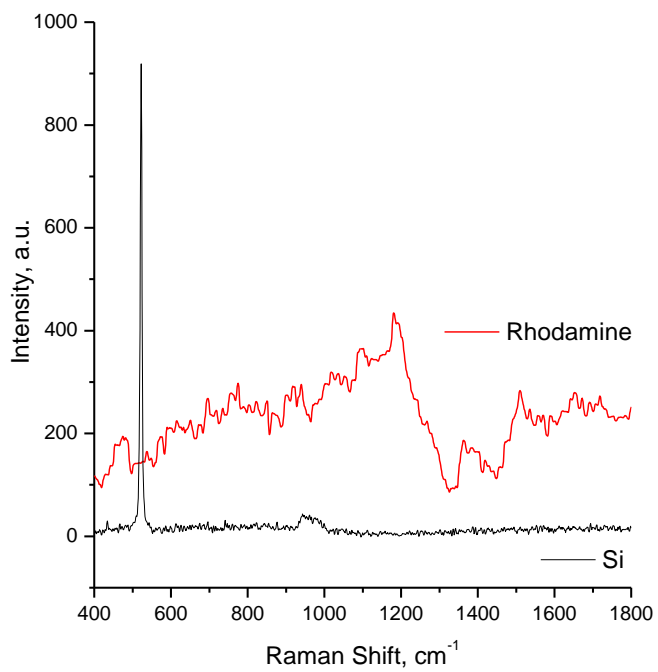

a)

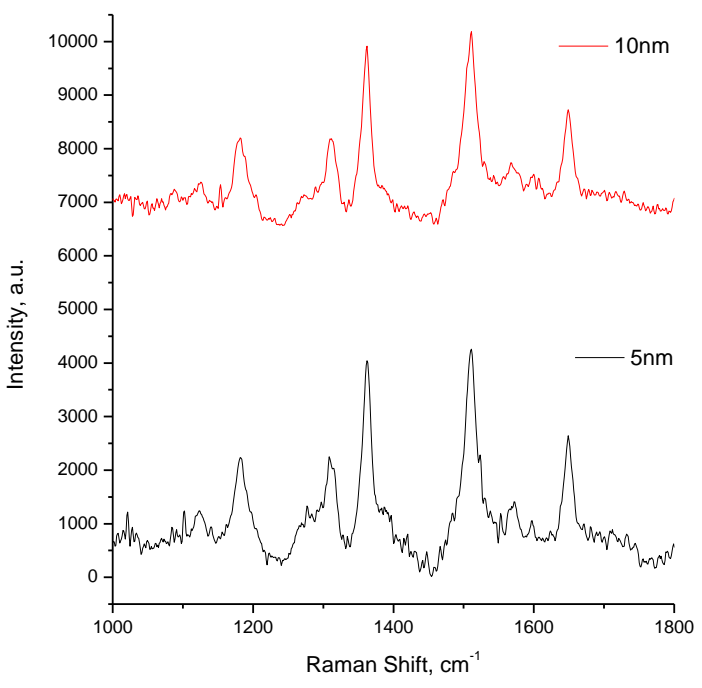

b)

Figure 2. Raman spectra of pure silicon and Rhodamine $6 \mathrm{G}$ a) and Raman spectra of SERS substrate and Rhodamine. 